
combination analysis. pathogens.

\title{
Identification of a peroxidase inhibitor that enhances kanamycin activity
}

\author{
Zhen Hui ${ }^{1} \dagger$, Shiyi $\mathrm{Liu}^{1} \dagger$, Ruiqin $\mathrm{Cui}^{1}$, Biao Zhou ${ }^{1}$, Chunxia $\mathrm{Hu}^{1}$, Min Zhang ${ }^{1}$, Qiuyang
} Deng ${ }^{1}$, Shumin Cheng ${ }^{1}$, Yutian $\mathrm{Luo}^{2}$, Huaisheng $\mathrm{Chen}^{2}$, Jinsong $\mathrm{Wu}^{3}$, Yuemei $\mathrm{Lu}^{3}$, Xueyan Liu $^{2}$, Lingyun Dai ${ }^{1}$, Wei Huang ${ }^{1 *}$

${ }^{1}$ Bacteriology \& Antibacterial Resistance Surveillance Laboratory, Shenzhen Institute of Respiratory Diseases, Shenzhen People's Hospital (The Second Clinical Medical College, Jinan University; The First Affiliated Hospital, Southern University of Science and Technology), Shenzhen 518020, Guangdong, China; ${ }^{2}$ Intensive Care Unit, Shenzhen People's Hospital(The Second Clinical Medical College, Jinan University; The First Affiliated Hospital, Southern University of Science and Technology), Shenzhen 518020, Guangdong, China; ${ }^{3}$ Department of Clinical Laboratory, Shenzhen People's Hospital (The Second Clinical Medical College, Jinan University; The First Affiliated Hospital, Southern University of Science and Technology), Shenzhen 518020, Guangdong, China; *Corresponding author. E-mail: whuang_sz@163.com $\dagger$ Joint first authors.

Background: The threat of antimicrobial resistance calls for more efforts in basic science, drug discovery, and clinical development, particularly gram-negative carbapenem-resistant

Objectives and methods: Whole-cell-based screening was performed to identify novel antibacterial agents against Acinetobacter baumannii ATCC19606. Spontaneously resistant mutant selection, whole-genome sequencing, and surface plasmon resonance were used for target identification and confirmation. Checkerboard titration assay was used for drug 
30 Results: A small molecule named 6D1 with the chemical structure of 6-

31 fluorobenzo[d]isothiazol-3(2H)-one was identified and exhibited activity against $A$.

32 baumannii ATCC19606 strain (minimal inhibitory concentration, MIC $=1 \mathrm{mg} / \mathrm{L}$ ). The

33 mutation in the plasmid-derived $\operatorname{ohr} B$ gene that encodes a peroxidase was identified in

34 spontaneously resistant mutants. Treatment of the bacteria with 6D1 resulted in increased

35 sensitivity to peroxide such as tert-butyl hydroperoxide. The binding of 6D1 and OhrB was

36 confirmed by surface plasmon resonance. Interestingly, the MIC of kanamycin against

37 spontaneously resistant mutants decreased. Finally, we identified the effect of 6D1 on

38 enhancing the antibacterial activity of kanamycin, including New Delhi metallo- $\beta$-lactamase

39 (NDM-1)-producing carbapenem-resistant Klebsiella pneumoniae, but not in strains carrying

40 kanamycin resistance genes.

42 Conclusions: In this study, we identified a peroxidase inhibitor that suppresses the growth of

A. baumannii and enhances the antibacterial activity of kanamycin. We propose that peroxidase may be potentially used as a target for kanamycin adjuvant development.

\section{Introduction}

47 Gram-negative pathogens such as Acinetobacter baumannii, Klebsiella pneumoniae, and Pseudomonas aeruginosa have become resistant to almost all commonly used antimicrobial agents, including aminoglycosides, quinolones, and broad-spectrum $\beta$-lactams. Overall, for

50 instance, approximately $45 \%$ of all global $A$. baumannii isolates are considered multidrug51 resistant (MDR) (bacteria resistant to more than three antibiotic classes). ${ }^{1,2}$

52 With the emergence of carbapenem-resistant gram-negative pathogens such as $A$. baumannii (CRAB), tigecycline and polymyxin-class antibiotics are the only currently

54 available treatment options. ${ }^{3}$ However, treatment outcomes of tigecycline have been 
hampered by the low serum concentrations of the drug in the approved dosing regimen and the low penetration in the epithelial lining fluid (ELF) of mechanically ventilated patients. ${ }^{4}$

The resistance of polymyxin-class antibiotics as well as nephrotoxicity and neurotoxicity are the major factors that limit the usage of polymyxin. Previous clinical observations showed that the rates of nephrotoxicity occurred in approximately $60 \%$ of patients who received colistin or polymyxin B therapy. ${ }^{5-7}$ Eravacycline, cefiderocol, and plazomicin seem to be promising new agents against $A$. baumannii. However, evaluation of their position in clinical practice and particularly in ventilator-associated pneumonia (VAP) has not been performed to date. $^{8,9}$

The present clinical pipeline does not meet current needs, and thus more investment is required in basic science, drug discovery, and clinical development, particularly gramnegative carbapenem-resistant pathogens, including CRAB. ${ }^{10}$ Therefore, we launched a whole cell-based screening program for A. baumannii. Here, we report the discovery of compound 6D1 that exhibits anti-A. baumannii activity. In addition, we show that enhancement effect of 6D1 on the antibacterial efficacy of kanamycin through the inhibition of plasmid-derived OhrB.

\section{Materials and methods}

\section{Bacterial strains, growth conditions, reagents, and screening strategy}

A. baumannii, K. pneumoniae, and P. aeruginosa were grown in liquid broth (LB) medium or LB agar. Antibiotic (purchased from Sigma-Aldrich, USA) solutions were prepared at a concentration of $1 \mathrm{mg} / \mathrm{mL}$ in distilled water or $100 \%$ dimethylsulfoxide (DMSO), filtersterilized, and frozen at $-20^{\circ} \mathrm{C}$ until use. The compounds to be screened were dissolved in $100 \%$ DMSO and stored as frozen stocks at a concentration of $1 \mathrm{mg} / \mathrm{mL}$.

We sought anti-A. baumannii compounds by testing compounds for inhibition of $A$. 
baumannii ATCC19606. A whole-cell assay was used because of its ability to concurrently assess multiple targets. Compounds were prepared in 96-well plates at a concentration of 10 $\mathrm{mg} / \mathrm{L}$ in $50 \mu \mathrm{L} \mathrm{LB}$ broth. A $50-\mu 1$ aliquot of each bacteria culture was then added to each well of the 96-well plate at an $\mathrm{OD}_{600}=0.006$. The plates were incubated overnight at $37^{\circ} \mathrm{C}$, and the primary active hits were filtered by achieving at least $90 \%$ of bacterial growth inhibition using Cell Counting Kit-8 (MCE, USA). Subsequently, two-fold serial dilutions of primary hits were prepared for the determination of minimal inhibitory concentration (MIC, defined as the lowest concentration of compound that inhibited $90 \%$ of bacterial growth). Compounds with an $\mathrm{MIC} \leq 1 \mathrm{mg} / \mathrm{L}$ were selected for further investigation.

\section{Spontaneously resistant mutant selection}

Spontaneously resistant mutants were selected via stepwise exposure to increasing concentrations of the compounds. An aliquot of mid-log phase $\left(\mathrm{OD}_{600}=0.6\right)$ bacterial culture (1 mL) was added to $2 \mathrm{~mL}$ of medium containing serial increasing concentrations of 6D1 until no growth was observed. The bacteria that survived in culture were spread onto agar plates containing the corresponding concentrations of the 6D1 compound. All colonies that originated from different plates and represent independent biological events were subjected to whole-genome sequencing (WGS). The resistance phenotype to the compound was confirmed by testing for a shift in MIC values.

\section{WGS}

101 Genomic DNA was extracted from each isolate using a gram-negative bacterial genome extraction kit (Tiangen, China). Whole-genome fragment libraries were prepared using a paired-end sample preparation kit (Illumina, USA). The genomes were sequenced using

104 Illumina HiSeq 2500 platform (Illumina, USA) and assembled with de novo SPAdes Genome 
105 Assembler (version 3.12.0). ${ }^{11}$ The resulting reads were mapped to the A. baumannii 106 ATCC19606 reference genome, and mutations were identified using Snippy

107 (https://github.com/tseemann/snippy).

Effect of compounds on the tolerance of bacteria to peroxides

110 The effects on the tolerance of bacteria to peroxides was determined by testing for shifts in 111 MIC of tert-butyl hydroperoxide ( $t$-BHP), cumene hydroperoxide (CHP), and hydrogen 112 peroxide $\left(\mathrm{H}_{2} \mathrm{O}_{2}\right)$ in the presence of the compound.

\section{Protein expression and purification}

115 The cDNA encoding for full-length OhrB was chemically synthesized with codon optimization for expression in E. coli. Vector pET28b was used for protein expression. The

117 plasmid pET28b-ohrB was then transformed into competent BL21 strain cells. The BL21 118 cells carrying the aforementioned plasmid were grown in $\mathrm{LB}$ medium at $37^{\circ} \mathrm{C}$ to an $\mathrm{OD}_{600}$ of 119 0.4. The cell cultures were then supplemented with $0.5 \mathrm{mM}$ isopropyl $\beta$-D-1thiogalactopyranoside (IPTG). The induced cells were further grown at $16^{\circ} \mathrm{C}$ with shaking at $220 \mathrm{rpm}$ overnight to induce the expression of the recombinant protein. The supernatant of the lysed cells was loaded onto Ni-NTA columns (Qiagen, Germany), and

124 the proteins were further purified by a gel-filtration column (GE Healthcare, USA) with a gel-filtration buffer [100 mM NaCl, $10 \mathrm{mM}$ Tris- $\mathrm{HCl}(\mathrm{pH} 7.5)$ and $1 \mathrm{mM}$ DTT]. The protein concentration was determined using the Bradford method.

\section{Surface plasmon resonance (SPR) experiment}

129 OhrB were covalently immobilized to a sensor chip CM5 (29-1049-88, Sweden) by means of 
amino coupling. The running buffer used in the experiment contains $20 \mathrm{mM}$ HEPES pH 7.5, $150 \mathrm{mM} \mathrm{NaCl}, 0.05 \%$ Tween 20, 0.1\% DMSO, and the 6D1 compound was also dissolved in the running buffer. The sensor chip was washed with running buffer between each concentration. Reference runs were performed with blank (sensor chip only) and active

134 (sensor chip with OhrB only) channel on the same sensor chip. The assay curves were constructed using serial concentrations of $6 \mathrm{D} 1$ of $7.5,15,30,60$, and $120 \mu \mathrm{M}$. The kinetic parameters of the interaction and the affinity constants were calculated using Biacore T200 evaluation software.

\section{Checkerboard titration assay}

140 Drug interactions between 6D1 and the bactericidal drugs were performed using a 141 chequerboard titration assay. ${ }^{12}$ The fractional inhibitory concentration (FIC) was calculated

142 using the following formula: (MIC of drug A or B in combination)/(MIC of drug A or B 143 alone). The fractional inhibitory concentration index (FICI) was determined by adding the 144 two FICs. Synergy, antagonism, and no interaction were defined as FICI $\leq$ 0.5, FICI > 4.0, 145 and FICI $=0.5-4.0$, respectively. ${ }^{12}$

147 Results

\section{$148 \quad$ In vitro activity of 6D1}

149 We identified an active hit named 6D1 (MIC $=1 \mathrm{mg} / \mathrm{L}$ ) with the structure of 6150 fluorobenzo[d]isothiazol-3(2H)-one, which is similar to 1, 2-benzisothiazolin-3-one (BIT) 151 and an antifungal drug ticlatone (Figure 1). A moderate antibacterial activity of 6D1 was also 152 observed in S. aureus (MIC = $2.5 \mathrm{mg} / \mathrm{L}$ ), but not in $K$. pneumoniae, and P. aeruginosa (MIC $153 \geq 5 \mathrm{mg} / \mathrm{L}$ ). Unexpectedly, MICs of 6D1 were high in CRAB clinical isolates (MIC = 5-10 $154 \mathrm{mg} / \mathrm{L})($ Table 1$)$. 


\section{OhrB mutations confer resistance to 6D1}

157 To identify the target of 6D1, we obtained two 6D1 spontaneously resistant strains (3M and $5 \mathrm{M})$ from independent cultures with bacterial growth in LB broth containing $10 \times$ MIC (10 $\mathrm{mg} / \mathrm{L}$ ) of 6D1. An increase in MIC indicated the resistance phenotype of 3M and 5M to 6D1

160 (Table 1). Compared with the wild-type (WT) parent strain A. baumannii ATCC19606, mutations located in plasmid (pMAC)-derived $o h r B$ were identified both in $3 \mathrm{M}$ and $5 \mathrm{M}$ strains, resulting in the conversion of arginine at the position 15 (Arg15) of OhrB (Table 2). Molecular dynamics simulations and in silico mutagenesis indicated that the corresponding Arg19 in Ohr from Xylella fastidiosa contributed to the stabilization of XfOhr in the closed state, suggesting that the mutations in $3 \mathrm{M}$ and $5 \mathrm{M}$ probably affect the function of OhrB. ${ }^{13}$

\section{D1 reduces the tolerance of bacteria to peroxides}

Ohr was first described in Xanthomonas campestris. It has since been found in a number of bacterial species. ${ }^{14,15}$ Owing to the Cys-based, thiol-dependent peroxidase activity, Ohr plays a central role in bacterial responses against fatty acid hydroperoxides and peroxynitrite, thus resulting in an "organic hydroperoxide resistance" phenotype. ${ }^{16}$ Table 3 shows that the MICs of $t$-BHP, CHP, and $\mathrm{H}_{2} \mathrm{O}_{2}$ in A. baumannii ATCC19606 were at least eight-fold lower when in the presence of $2.5 \mathrm{mg} / \mathrm{L}$ of $6 \mathrm{D} 1$. The magnitude of MIC reduction coincided with the

174 substrate preference of $\mathrm{Ohr}\left(\mathrm{H}_{2} \mathrm{O}_{2} \ll<<\mathrm{CHP}<t\right.$-BHP $)$ as previously reported. ${ }^{16}$ In contrast, in $3 \mathrm{M}$ strain, the MICs of $t$-BHP, CHP, and $\mathrm{H}_{2} \mathrm{O}_{2}$ were almost not affected by the presence of 6D1; whereas in 5M strain, the MICs of $t$-BHP, CHP, and $\mathrm{H}_{2} \mathrm{O}_{2}$ were reduced, indicating that 6D1 could still affect the function of OhrB in 5M strain. However, the MICs of $t$-BHP and $\mathrm{H}_{2} \mathrm{O}_{2}$ in $3 \mathrm{M}$ or $5 \mathrm{M}$ strains were slightly lower than the WT, suggesting that the $o h r B$ mutation resulted in reduced tolerance to peroxides. Moreover, growth retardation was also observed 
in 3M and 5M strains, but the growth of 3M strain was not affected by 6D1 (Figure 2).

\section{MICs of bactericidal drugs in $3 \mathrm{M}$ and $5 \mathrm{M}$ strains}

With aim to identify the effects of OhrB protein function on the activity of bactericidal agents, we tested the MICs of a handful of bactericidal drugs in the $3 \mathrm{M}, 5 \mathrm{M}$, and WT strains. Compared with the WT, the MICs of kanamycin in $3 \mathrm{M}$ and $5 \mathrm{M}$ strains decreased by at least 2-fold, thereby suggesting the association between $\mathrm{OhrB}$ function and kanamycin activity (Table 4).

\section{Drug combinations study}

190 The observed changes of MIC for kanamycin in 3M and 5M suggest that 6D1 probably enhances antibacterial activity. Therefore, we used a checkerboard titration assay to identify the drug interaction of 6D1 and kanamycin in different species. Table 5 revealed a synergistic effect between 6D1 and kanamycin in K. pneumonae and A. baumannii (FICI = 0.5). Clinical

194 isolates of carbapenem-resistant K. pneumonae (CRKP) were also selected to test the activity of 6D1 and kanamycin combination. The results showed that 6D1 did not reverse the antibacterial activity of kanamycin in CRKP containing the kanamycin resistance gene. However, we found that 6D1 enhanced the activity of kanamycin against a strain of CRKP that harbored the NDM-1 gene (Table 5, Table S1).

\section{The interaction between 6D1 and OhrB}

To characterize the binding of 6D1 and OhrB, we first obtained the purified His-tag-fused recombinant OhrB protein. The interaction between 6D1 and OhrB was confirmed by SPR analysis. It demonstrated the binding of 6D1 to $\mathrm{OhrB}$, with an association rate constant of $k_{a}$ 
$2042.33 \times 10^{3} \mathrm{M}^{-1} \mathrm{~s}^{-1}$, a dissociation rate constant $k_{d} 2.28 \times 10^{-3} \mathrm{~s}^{-1}$, and an equilibrium

205 dissociation constant $K_{D} 9.79 \times 10^{-6} \mathrm{M}$ (Figure 3).

206

207 Discussion

208 Bacteria have evolved complex mechanisms to detoxify reactive oxygen species and thus strictly control hydroperoxide levels. A 9,540-bp plasmid pMAC carried by A. baumannii ATCC19606 that contains an OhrB coding region conferred bacterial resistant to organic

211 peroxide-generating compounds CHP and $t$-BHP was reported in $2006 .{ }^{17}$ In this study, a 212 whole-cell assay revealed a compound 6D1, which imparted inhibitory effects on $A$. 213 baumannii ATCC19606 and had a similar structure to BIT and the antifungal ticlatone. The mutation site of the spontaneously resistant mutant suggested that the target of 6D1 was pMAC-derived OhrB. A previous study suggested that cellular thiol groups are major targets of BIT. ${ }^{18}$ Therefore, it provides a rationale that $6 \mathrm{D} 1$ acts on the thiol groups of OhrB. This is concordant with our result that 6D1 sensitizes A. baumannii ATCC19606 to CHP and $t$-BHP.

218 Because most clinical isolates do not contain pMAC, this can explain why 6D1 is ineffective in clinical isolates.

220

In addition to developing antibiotics with new chemical structures and acting mechanisms, antibiotic adjuvants offer an alternative approach to combat resistance. ${ }^{19}$ In this study, 6D1 was found to impart an inhibitory effect on $\mathrm{OhrB}$, and thus it is reasonable to use this as an adjuvant in combination with other antibiotics that induce bacteria to produce hydroperoxides.

224 In addition, a previous study showed that all bactericidal antibiotics induce protective responses to reactive oxygen species. ${ }^{20}$ This suggests the potential of $6 \mathrm{D} 1$ as an adjuvant for bactericidal drugs. However, our data showed that 6D1 only enhances the activity of the aminoglycoside drug kanamycin but not others. This may be related to the reactivity order of

228 Ohr to different peroxides, in which it mainly modulates the levels of fatty acid 
229 hydroperoxides and peroxynitrite. ${ }^{16}$ Because the effect of 6D1 is achieved by inhibiting OhrB,

230 it is not surprising that 6D1 was not effective on drug-resistant strains that harbored

231 kanamycin resistance genes such as the 16s rRNA methylase enzyme rmtB. Notably, the

232 combination of 6D1 and kanamycin was effective on the CRKP strain carrying NDM-1. A

233 recent study has shown that in Northeast China, the aminoglycoside resistance gene $r m t B$ was

234 detected in $96.61 \%$ of KPC-2-producing CRKP and in 21.74\% of NDM-1-producing CRKP,

235 indicating the potential combinative application of kanamycin and a peroxidase inhibitor such

236 as $6 \mathrm{D} 1$ in about $80 \%$ of NDM-1-producing CRKP. ${ }^{21}$

237 The clinical use of kanamycin has been limited by its well-known toxicity and side effects

238 such as ototoxicity. Our study revealed the feasibility of enhancing the activity of kanamycin

239 by inhibiting the detoxification ability of bacteria to peroxides, thereby providing a new

240 target and strategy for the development of kanamycin enhancers in the near future.

241

\section{Funding}

243 The International Collaborative Research Fund (GJHZ20180413181716797) and Free

244 Inquiry Fund (JCYJ20180305163929948) of Shenzhen Science and Technology Innovation

245 Commission supported this study.

\section{Transparency declarations}

248 None to declare.

\section{References}

2511 U.S. Department of Health and Human Services: Centers for Disease Control and

252 Prevention. Antibiotic Resistant Threats In The United States; 2019. 
2 Magill SS, Edwards JR, Bamberg W et al. Multistate point-prevalence survey of health care-associated infections. N Engl J Med. 2014; 370:1198-208.

3 Garnacho-Montero J, Dimopoulos G, Poulakou G et al. Task force on management and prevention of Acinetobacter baumannii infections in the ICU. Intensive Care Med. 2015; 41:2057-75.

4 Giamarellou H, Poulakou G. Pharmacokinetic and pharmacodynamic evaluation of tigecycline. Expert Opin Drug Metab Toxicol. 2011; 7:1459-70.

5 Xie R, Zhang XD, Zhao Q et al. Analysis of global prevalence of antibiotic resistance in Acinetobacter baumannii infections disclosed a faster increase in OECD countries. Emerg Microbes Infect. 2018; 7:31.

6 Qureshi ZA, Hittle LE, O'Hara JA et al. Colistin-resistant Acinetobacter baumannii: beyond carbapenem resistance. Clin Infect Dis. 2015; 60:1295-303.

7 Hartzell JD, Neff R, Ake J et al. Nephrotoxicity associated with intravenous colistin (colistimethate sodium) treatment at a tertiary care medical center. Clin Infect Dis. 2009; 48:1724-8.

8 Connors KP, Housman ST, Pope JS et al. Phase I, open-label, safety and pharmacokinetic study to assess bronchopulmonary disposition of intravenous eravacycline in healthy men and women. Antimicrob Agents Chemother. 2014; 58:21138.

9 Poulakou G, Lagou S, Karageorgopoulos DE et al. New treatments of multidrugresistant Gram-negative ventilator-associated pneumonia. Ann Transl Med. 2018; 6:423. analysis of the antibacterial clinical development pipeline, including tuberculosis. World 

NC-SA 3.0 IGO

11 Bankevich A, Nurk S, Antipov D et al. SPAdes: a new genome assembly algorithm and its applications to single-cell sequencing. J Comput Biol. 2012; 19:455-77.

12 Odds FC. Synergy, antagonism, and what the chequerboard puts between them. $J$ Antimicrob Chemother. 2003; 52:1.

13 Piccirillo E, Alegria TGP, Discola KF et al. Structural insights on the efficient catalysis of hydroperoxide reduction by Ohr: Crystallographic and molecular dynamics approaches. PLoS ONE 2018;13: e0196918.

14 Mongkolsuk S, Praituan W, Loprasert S et al. Identification and characterization of a new organic hydroperoxide resistance (ohr) gene with a novel pattern of oxidative stress regulation from Xanthomonas campestris pv. phaseoli. J Bacteriol. 1998; 180:2636-43.

15 Atichartpongkul S, Loprasert S, Vattanaviboon $\mathrm{P}$ et al. Bacterial Ohr and OsmC paralogues define two protein families with distinct functions and patterns of expression. Microbiology (Reading). 2001; 147:1775-82.

16 Alegria TG, Meireles DA, Cussiol JR et al. Ohr plays a central role in bacterial responses against fatty acid hydroperoxides and peroxynitrite. Proc Natl Acad Sci U S A. 2017; 114:E132-41.

17 Dorsey CW, Tomaras AP, Actis LA. Sequence and organization of pMAC, an Acinetobacter baumannii plasmid harboring genes involved in organic peroxide resistance. Plasmid. 2006; 56:112-23.

18 Fuller SJ, Denyer SP, Hugo WB et al. The mode of action of 1, 2-benzisothiazolin-3-one on Staphylococcus aureus. Letters in applied microbiology. 1985; 1: 13-5. 
30019 Wright GD. Antibiotic Adjuvants: Rescuing Antibiotics from Resistance [published 301 correction appears in Trends Microbiol. 2016 Nov;24(11):928]. Trends Microbiol. 2016; 24:862-71.

30320 Kohanski MA, Dwyer DJ, Hayete B et al. A common mechanism of cellular death induced by bactericidal antibiotics. Cell. 2007; 130:797-810.

30521 Lin L, Xiao GX, Wang XN et al. In Vitro Antimicrobial Susceptibility Differences Between Carbapenem-Resistant KPC-2-Producing and NDM-1-Producing Klebsiella pneumoniae in a Teaching Hospital in Northeast China. Microbial Drug Resistance. 
309 Table 1. MICs of 6D1 that inhibited 90\% of the growth of different bacterial strains

Strain $\quad$ MIC (mg/L)

Acinetobacter baumannii ATCC19606

$3 \mathrm{M}^{*}$

$5 \mathrm{M}^{*}$

Klebsiella pneumoniae ATCC13883

Pseudomonas aeruginosa ATCC27853

CRAB-40178

CRAB-42264

CRAB-39713 ${ }^{\#}$

CRAB-41362 ${ }^{\#}$

CRAB-39351"

\section{1}

10

10

10

80

5

5

10

10

10

310 *Spontaneously resistant mutants of A. baumannii ATCC19606 against 6D1

311 \#Antimicrobial resistance genes were shown in Supplementary Table S1.

312 CRAB, carbapenem-resistant A. baumannii. 
Table 2. Whole-genome sequencing identified polymorphisms within spontaneously resistant mutants of A. baumannii ATCC19606 against 6D1

\begin{tabular}{|c|c|c|c|c|c|c|c|}
\hline $\begin{array}{c}\text { Position of } A . \\
\text { baumannii } 19606\end{array}$ & Reference & $3 \mathrm{M}$ & $5 \mathrm{M}$ & $\begin{array}{l}\text { Locus } \\
\text { (DJ41_) }\end{array}$ & Gene & Product & Effect of mutation \\
\hline $\begin{array}{c}\text { NZ_KL810966.1: } \\
1913491\end{array}$ & $\mathrm{G}$ & $\mathrm{T}$ & - & RS13465 & - & LysR family transcriptional regulator & Asn272Lys \\
\hline $\begin{array}{c}\text { NZ_KL810966.1: } \\
2417811\end{array}$ & $\mathrm{G}$ & - & A & RS0104185 & - & hypothetical protein & Gly951Asp \\
\hline $\begin{array}{c}\text { NZ_KL810967.1: } \\
13770\end{array}$ & $\mathrm{C}$ & - & $\mathrm{T}$ & RS22870 & $o h r$ & organic hydroperoxide resistance protein & Arg15His \\
\hline $\begin{array}{c}\text { NZ_KL810967.1: } \\
13771\end{array}$ & $\mathrm{G}$ & A & - & RS22870 & $o h r$ & organic hydroperoxide resistance protein & Arg15Cys \\
\hline
\end{tabular}


315 Table 3. Effect of 6D1 on the susceptibility to peroxides

\begin{tabular}{ccccccc}
\hline $\begin{array}{c}\text { Peroxide } \\
(\mathrm{mM})\end{array}$ & Wild-type & Wild-type $^{\mathrm{a}}$ & $3 \mathrm{M}$ & $3 \mathrm{M}^{\mathrm{a}}$ & $5 \mathrm{M}$ & $5 \mathrm{M}^{\mathrm{a}}$ \\
\hline$t$-BHP & 0.2 & $<=0.005$ & 0.1 & 0.1 & 0.1 & 0.1 \\
$\mathrm{CHP}$ & 0.2 & $<=0.01$ & 0.2 & 0.2 & 0.2 & 0.08 \\
$\mathrm{H}_{2} \mathrm{O}_{2}$ & 0.8 & $<=0.1$ & 0.5 & 0.4 & 0.6 & 0.3 \\
\hline
\end{tabular}

$316{ }^{\mathrm{a}}$ The MICs of peroxides in the presence of $2.5 \mathrm{mg} / \mathrm{L}$ of $6 \mathrm{D} 1$.

$317 t$-BHP, tert-butyl hydroperoxide; CHP, cumene hydroperoxide; $\mathrm{H}_{2} \mathrm{O}_{2}$, hydrogen peroxide. 
318 Table 4. MICs of different classes of antibiotics that inhibited 90\% of bacterial growth

\begin{tabular}{cccc}
\hline Antibiotic & Wild-type $(\mathrm{mg} / \mathrm{L})$ & $3 \mathrm{M}(\mathrm{mg} / \mathrm{L})$ & $5 \mathrm{M}(\mathrm{mg} / \mathrm{L})$ \\
\hline Ceftazidime & 8 & 8 & 8 \\
Meropenem & 4 & 4 & 4 \\
Kanamycin & 16 & 4 & 8 \\
Ofloxacin & 0.25 & 0.25 & 0.25 \\
Polymyxin B & 2 & 2 & 2 \\
Tetracycline & 0.3125 & 0.625 & 0.625 \\
Rifampicin & 1.56 & 1.56 & 1.56 \\
Chloramphenicol & 64 & 64 & 64
\end{tabular}


Table 5. Potency of 6D1 in combination with kanamycin against different species

\begin{tabular}{|c|c|c|c|c|}
\hline & MIC of 6D1 & & Fold change & \\
\hline Strain & (mg/L) & MIC of Kanamycin $(\mathrm{mg} / \mathrm{L})$ & $(\mathrm{mg} / \mathrm{L})^{\#}$ & FICI \\
\hline A. baumannii 19606 & 1 & 16 & $4(0.25)$ & 0.5 \\
\hline $3 \mathrm{M}$ & 10 & 4 & $2(0.625)$ & 0.5625 \\
\hline $5 \mathrm{M}$ & 10 & 8 & $4(2.5)$ & 0.5 \\
\hline K. pneumoniae 13883 & 10 & 8 & $4(2.5)$ & 0.5 \\
\hline CRKP-34978* & 20 & 16 & $4(5)$ & 0.5 \\
\hline CRKP-36472* & 10 & $>256$ & - & - \\
\hline CRKP-38218* & 10 & $>256$ & - & - \\
\hline CRKP-42644* & 20 & $>256$ & - & - \\
\hline CRKP-40847* & 10 & $>256$ & - & - \\
\hline
\end{tabular}

321 *Antimicrobial resistance genes were shown in Supplementary Table S1.

322 \#The concentration of 6D1 used in the combination. 
Table S1. Antimicrobial resistance genes in clinical isolates

Clinical isolate Antimicrobial resistance genes

CRAB-40278 aph(6)-Id, blaOXA-23, blaOXA-88, blaTEM-2, $m p h(E), \operatorname{msr}(E), \operatorname{strA}, \operatorname{sul} 2, \operatorname{tet}(B)$

CRAB-42264 aph(3')-Ia, aph(6)-Id, armA, blaADC-25, blaOXA-23, blaOXA-66, blaTEM-1D, mph(E), msr(E), strA, sul2, tet(B)

CRAB-39713 aph(6)-Id, armA, blaADC-25, blaOXA-23, blaOXA-66, blaTEM-1D, mph(E), msr(E), strA, tet(B)

CRAB-41362 aac(6')-Ib, aac(6')-Ib-cr, aadA1, aph(3')-Ia, aph(6)-Id, armA, blaADC-25, blaOXA-23, blaOXA-80, blaTEM-1D, catB8, $m p h(E), \operatorname{msr}(E), \operatorname{str} A, \operatorname{sull}, \operatorname{sul} 2, \operatorname{tet}(B)$

CRAB-39351 aph(3')-Ia, aph(6)-Id, armA, blaADC-25, blaOXA-23, blaOXA-66, blaTEM-1D, mph(E), msr(E), strA, tet(B)

CRKP-34978 aadA1, blaNDM-1, blaSHV-155, dfrA15, fosA, oqxA, oqxB, sul1

CRKP-36472 aac(6')-Ib-cr, aac(6')-Ib-Hangzhou, blaIMP-4, blaLEN22, blaSFO-1, catB8, mph(A), mph(E), msr(E), qnrS1, sul1

CRKP-38218 aadA2, blaCTX-M-65, blaKPC-2, blaSHV-155, blaTEM-1B, fosA6, rmtB, sul1

CRKP-42644 aadA2, aph(3")-Ib, aph(6)-Id, blaCTX-M-65, blaKPC-2, blaSHV-11, blaTEM-1B, dfrA1, fosA3, fosA6, oqxA, oqxB, qnrS1, $r m t B, \operatorname{sull}, \operatorname{sul} 2, \operatorname{tet}(A)$ 
CRKP-40847 aac(3)-IId, aac(3)-IVa, aac(6')-Ib-cr, aac(6')-Ib-cr, aadA2, aadA5, aph(3")-Ib, aph(4)-Ia, aph(6)-Id, armA, ARR-3, blaIMP-

4, blaOXA-1, blaSFO-1, blaSHV-11, blaTEM-1B, catB4, dfrA1, dfrA12, floR, fosA6, mph(A), mph(E), msr(E), oqxA, oqxB, qnrB52, qnrS1, sul1, sul2 
325 Figure 1. Chemical structure of 6D1 and its analogues. (a) 6D1. (b) 1, 2-benzisothiazolin-3326 one (BIT). (c) Ticlatone.

327

328 Figure 2. Growth curve of wild-type Acinetobacter baumannii ATCC19606, 3M, and 5M in

329 the absence or presence of $0.25 \mathrm{mg} / \mathrm{L} 6 \mathrm{D} 1$.

330

331 Figure 3. Surface plasmon resonance analysis of the interaction between 6D1 and OhrB. The

$332 K_{D}$ values were determined from the ratio between the kinetic rate constants $\left(k_{a} / k_{d}\right)$. 


\section{Figure 1}

(a) $6 \mathrm{D} 1$<smiles>O=c1[nH]sc2cc(F)ccc12</smiles>

(b) BIT<smiles>O=c1[nH]sc2ccccc12</smiles>

(c) Ticlatone<smiles>O=c1[nH]sc2cc(Cl)ccc12</smiles> 


\section{Figure 2}

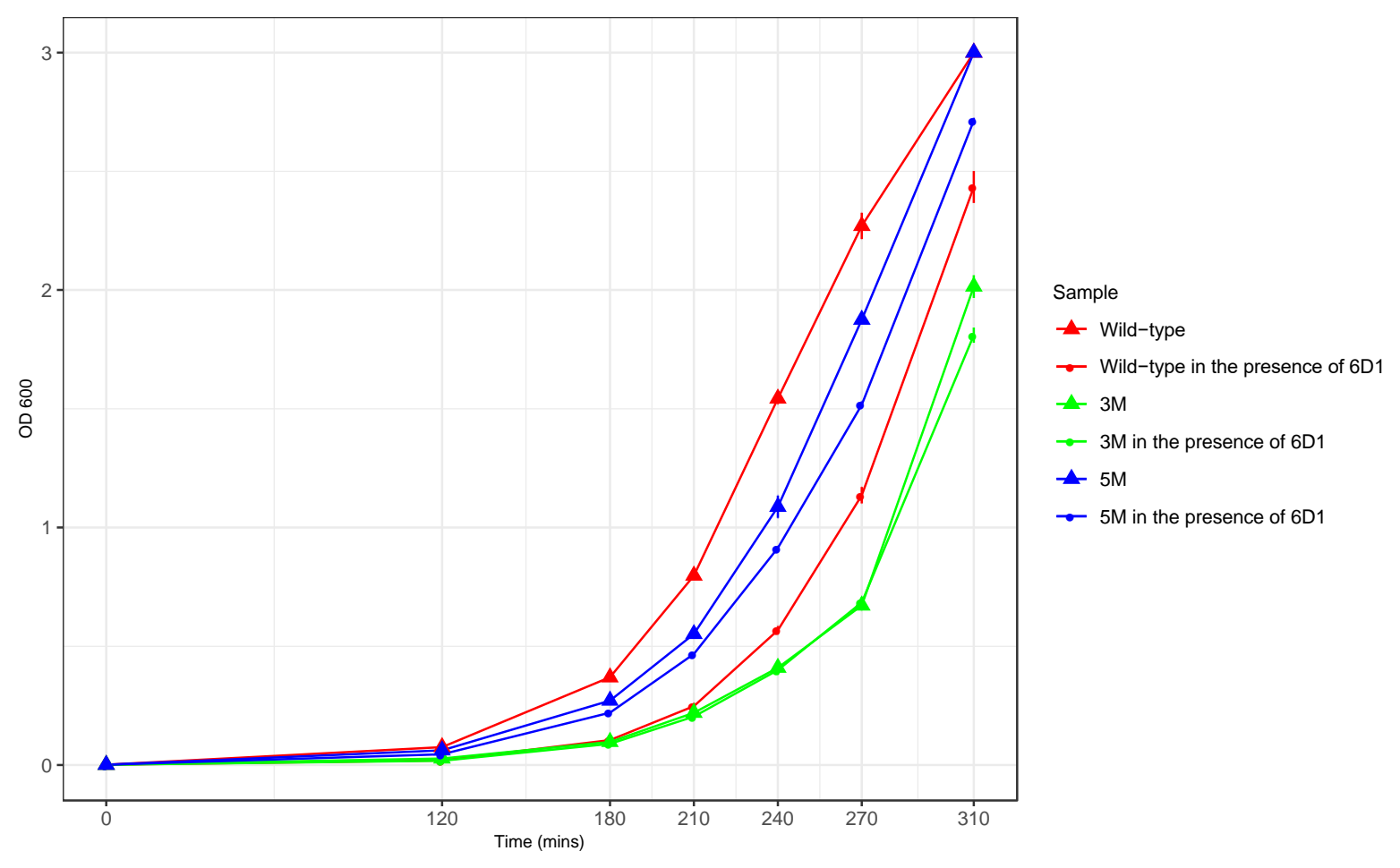




\section{Figure 3}

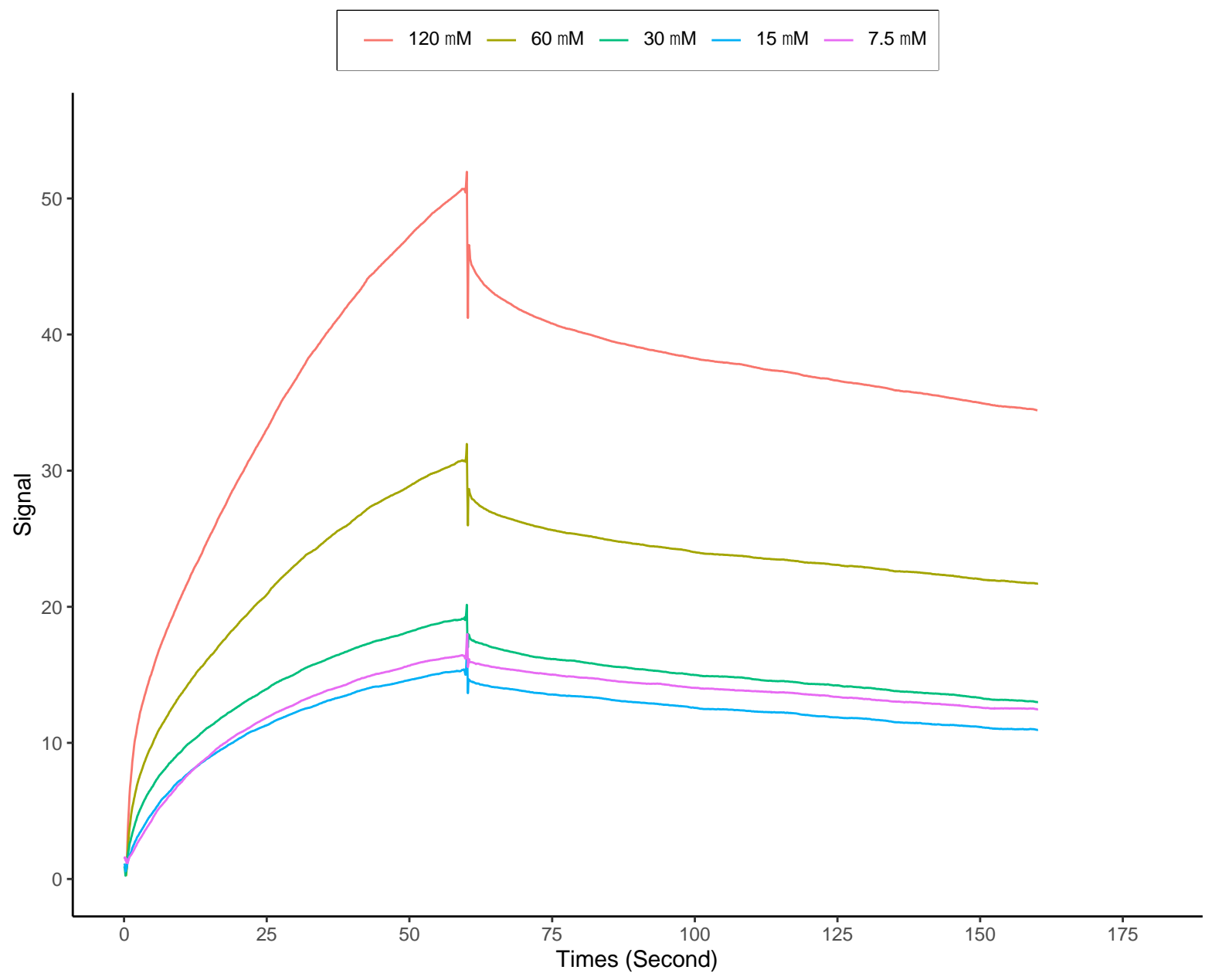

